\title{
Leistungserbringungsrecht als Kodifikationsproblem
}

\author{
Andreas Hänlein
}

$I$.

Maximilian Fuchs hat darauf aufmerksam gemacht, dass das Sachleistungsprinzip erst durch das Gesundheitsreformgesetz explizit im Gesetz, also im damals neuen SGB V, verankert wurde. Andererseits soll nach der zitierten Entscheidung des BSG seine Fortgeltung als übernormatives Grundprinzip des Rechts der gesetzlichen Krankenversicherung seit Inkrafttreten der RVO völlig unbestritten gewesen sein. ${ }^{1}$ Dies ist ein erstaunlich widersprüchlicher Befund. Man fragt sich, weshalb es nicht schon früher zu einer Kodifikation kam, insbesondere warum keine einschlägige Grundsatznorm Eingang in einen der Allgemeinen Teile des Sozialgesetzbuchs gefunden hatte.

Dieses Erstaunen möchte ich zum Anlass nehmen, über den kodifikatorischen $\mathrm{Zu}$ stand des Rechts der Leistungserbringung im Allgemeinen nachzudenken und zunächst einige einschlägige Befunde zu benennen.

\section{II.}

1. Ein erster Befund bezieht sich auf Regeln zum Leistungserbringungsrecht in den allgemeinen Teilen. Dort finden wir zwar nichts zum Sachleistungsprinzip. Das Leistungserbringungsrecht wurde jedoch nicht vergessen. So sind nach § 11 SGB I Dienst-, Sach- und Geldleistungen Gegenstand der sozialen Rechte. Nach $§ 1$ Abs. 2 SGB I soll das Recht des Sozialgesetzbuchs auch dazu beitragen, dass die zur Erfüllung der Aufgaben des Sozialgesetzbuchs erforderlichen sozialen Dienste und Einrichtungen rechtzeitig und ausreichend zur Verfügung stehen. Hierauf werden die Leistungsträger durch $\S 17$ Abs. 1 Nr. 2 SGB I dann nochmals ausdrücklich verpflichtet. In diesen Vorschriften thematisiert das Gesetz mithin die Verantwortung der Leistungsträger für die Infrastruktur sozialer Dienste. Einen Titel aber mit „Grundsätzen des Leistungserbringungs-

1 BSGE 69, 170, 173; dass das jedenfalls in den ersten Jahren der gesetzlichen Krankenversicherung so selbstverständlich nicht war, hat Fischer, Der Sachleistungsgrundsatz - ein unantastbares Urprinzip der GKV, SGb 2008, S. 461 ff., soeben nochmals deutlich gezeigt. Auch das BVerfG betont übrigens den Rang des Prinzips, wenn es einer eventuellen Abkehr vom Sachleistungsprinzip eine solch erhebliche Tragweite für das System der gesetzlichen Krankenversicherung beimisst, dass nur der Gesetzgeber selbst sie verantworten könne (BVerfGE 106, 275, 309). 
rechts“, etwa nach dem Vorbild der Grundsätze des Leistungsrechts in den §§ 38-59 SGB I, gibt es nicht. Das SGB I blickt fast ausschließlich auf das Sozialleistungsverhältnis. Die Kodifikation versteht Sozialleistungsrecht primär als Recht der Geldleistungen, auch wenn sie sich bemüht, die sozialen Dienstleistungen in Ansätzen zu thematisieren. ${ }^{2}$ Das SGB IV ist in diesem Zusammenhang völlig unergiebig. Im SGB X gibt es im Dritten Kapitel immerhin noch einen einschlägigen Paragraphen, wo es um die Zusammenarbeit der Leistungsträger mit Dritten geht: § 97 I SGB X spricht von der Gewährleistungsverantwortung der Leistungsträger, wenn diese Aufgaben von Dritten wahrnehmen lassen. ${ }^{3}$

2. Dies leitet über zu weiteren Befunden, die sich auf die besonderen Teile beziehen. Dort gibt es inzwischen eine große Zahl vergleichsweise geschlossener bereichsspezifischer Teil- oder Binnenkodifikationen, die teilweise auch im Bereich des Leistungserbringungsrechts die Gesetzesqualität verbessert haben, teilweise aber auch nicht.

a) $\mathrm{Zu}$ erwähnen ist zunächst die systematisierende Neukodifikation des Krankenversicherungsrechts in Form des SGB V durch das Gesundheitsreformgesetz ${ }^{4}$. Hinsichtlich der medizinischen Leistungen war das SGB V gewiss ein kodifikatorischer Fortschritt ${ }^{5}$, und zwar auch in puncto Leistungserbringungsrecht. Zu nennen ist hier außer den allgemeinen Regeln über Sachleistungen und Kostenerstattung ${ }^{6}$ vor allem das Kapitel über die Beziehungen der Krankenkassen zu den Leistungserbringern. Dieses Kapitel ist übersichtlich nach Leistungserbringern gegliedert, so dass man sich darin wohl besser zurechtfindet, als in den $\S \S 368-376 \mathrm{~b}$ RVO. Und es gibt einen Ansatz, allgemeine Aussagen vor die Klammer zu ziehen. Der Abstrahierungseifer des Gesetzgebers war freilich überschaubar, herausgekommen sind die $\S \S 69-71$ SGB V. Und: § 69 enthält eine Art Verlustliste, wenn es dort heißt, dass die Beziehungen zu den Krankenhäusern abschließend im Vierten Kapitel zuzüglich Krankenhausfinanzierungsgesetz, Krankenhausentgeltgesetz und einschlägiger Rechtsverordnungen geregelt sind. Auch im Detail verhindern Vorschriften wie diejenigen über die ärztliche Vergütung ( $§ 85$ ff. SGB V), dass Euphorie aufkommt.

b) $\mathrm{Zu}$ weiteren Binnenkodifikationen kam es u. a. in den Sicherungszweigen, in denen soziale Dienstleistungen eine besondere Rolle spielen. Hinsichtlich Pflege, Kinderund Jugendhilfe und Sozialhilfe verfügen wir heute über eigenständige Teilkodifikationen, die jeweils Abschnitte über die mit Leistungserbringern zu schließenden Verträge

2 Zacher, Das Vorhaben des Sozialgesetzbuchs, RsDE 47 (2002), S. 1 ff., 22 f.

3 Kritisch Giese, Zusammenarbeit der Leistungsträger und ihre Beziehungen zu Dritten nach dem Sozialgesetzbuch (SGB X 3. Kapitel), ZfF 1983, S. 97, 98: die Überschrift des Titels sei eine „überfreundliche gesetzgeberische Beschreibung für einige einseitige Pflichten“; anders Schellhorn, in: von Maydell/Schellhorn, GK-SGB X 3, Neuwied 1984, § 97 Rdnr. 7.

4 Gesundheitsreformgesetz (GRG) vom 20.12.1988, BGBl. I S. 2477; zur besonderen kodifikatorischen Aufgabe dieses Gesetzes z.B. Ebsen, in: von Maydell/Ruland/Becker (Hrsg.), Sozialrechtshandbuch, 4. Aufl. Baden-Baden 2008, § 15 Rdnr. 19.

$5 \mathrm{Zu}$ den Intentionen BT-Drs. 11/2237, S. 136.

6 BT-Drs. 11/2237, S. 146. 
enthalten. Für die Entwicklung dieser Regelungskomplexe war zunächst die Einführung der Pflegeversicherung wichtig. ${ }^{7}$ Bereits in den allgemeinen Vorschriften am Anfang des SGB XI kommen die Leistungserbringer vor (v. a. § 11 SGB XI), und im Kapitel über die Beziehungen der Pflegekassen zu den Leistungserbringern (§§ 69-81 SGB XI) sowie im kurioserweise eigenständigen Kapitel über die Pflegevergütung (§§ 82-92b SGB XI) findet sich ein ausgeprägtes und durchgeformtes Vertragsrecht der Leistungserbringung mit Regelungen über Leistungs- und Vergütungsvereinbarungen sowie Vorkehrungen für die Vertragshilfe durch Schiedsstellen.

Ganz ähnliche Vorschriften enthalten heute auch SGB VIII und SGB XII. Die Herausbildung dieser Vertragsrechte wurde 1993/1994 vorangetrieben im Zusammenhang mit der Abkehr vom Selbstkostendeckungsprinzip zugunsten prospektiv zu vereinbarender Vergütungen für Leistungserbringer im Sozialhilferecht ${ }^{8}$, die ihrerseits von den damals ins Auge gefassten Strukturen des Rechts der Pflegeversicherung beeinflusst war. ${ }^{9}$

Wenig später wurde in der jugendhilferechtlichen Praxis der Ruf laut, auch im SGB VIII ein am Sozialhilferecht orientiertes Vereinbarungssystem zur Vereinbarung prospektiver Leistungsentgelte einzuführen. Eine Arbeitsgruppe des Deutschen Vereins für öffentliche und private Fürsorge schlug die Regelung dieses Themenfeldes in einem eigenen Kapitel des SGB VIII vor. ${ }^{10}$ Dies führte letztlich zur Einführung der Vorschriften des SGB VIII über „Vereinbarungen über Leistungsangebote, Entgelte und Qualitätsentwicklung“, der §§ 78a-78g SGB VIII, die am 1.1.1999 in Kraft traten. ${ }^{11}$ Am selben Tag trat auch das Gesetz zur Reform des Sozialhilferechts in Kraft, das die sozialhilferechtlichen Regelungen über die mit Einrichtungsträgern zu treffenden Vereinbarungen erneut modifizierte ( $\S \S 93,93 a-93$ c BSHG). ${ }^{12}$ Heute finden sich die einschlägigen Vorschriften im Kapitel „Einrichtungen“ des SGB XII (§§ 75-81 SGB XII).

Hinsichtlich des Vereinbarungs- und Vergütungsrechts bei den sozialen Diensten nach SGB VIII, XI und XII kann man nun von einer dreifachen Parallelkodifikation sprechen, die auf das Herausdestillieren gemeinsamer, vor die Klammer zu stellender Regelungen verzichtet.

7 Gesetz zur sozialen Absicherung des Risikos der Pflegebedürftigkeit vom 26.5.1994, BGBl. I S. 1014; dazu der Regierungsentwurf vom 1.7.1993 BT-Drs. 12/5262.

8 Vgl. § 92 Abs. 2 BSHG i. d. F. des Zweiten Gesetzes zur Umsetzung des Spar-, Konsolidierungsund Wachstumsprogramms vom 21.12.1993, BGB1. I S. 2374; zur weiteren Entwicklung Schellhorn/Schellhorn, BSHG, 16. Aufl. Neuwied 2002, § 93, Rdnr. 1 ff.

9 Vgl. BT-Drs. 12/5510, S. 10 zur Abschaffung des Selbstkostendeckungsprinzips im Sozialhilferecht in Anlehnung an die Gesetzgebung für den Krankenhaussektor und mit Blick auf die geplanten Regeln für die Pflegeversicherung.

10 Entgeltregelung bei der Inanspruchnahme von Einrichtungen und Diensten in der Jugendhilfe, NDV 1997, S. 352.

11 Aufgrund des Zweiten Gesetzes zur Änderung des SGB XI vom 29.5.1998; dazu BT-Drs. 13/10330, S. 17.

12 Gesetz zur Reform des Sozialhilferechts vom 23.7.1996, BGBl. I S. 1088; dazu BT-Drs. 13/2440. 
c) In anderen Teilen des Sozialgesetzbuchs ist der Kodifikationsprozess bekanntlich in den letzten 20 Jahren ebenfalls vorangetrieben worden, allerdings ohne großen Ertrag - gemessen an Abstraktionsgrad oder Transparenz - hinsichtlich des Rechts der Leistungserbringung. Hier ist insbesondere an das SGB III zu denken. Dort finden sich interessante neue Formen der Leistungserbringung durch Dritte, etwa im Bereich der durch die Einführung der Instrumente des Bildungsgutscheins und der Zertifizierung von Weiterbildungsträgern vor kurzem erst dramatisch reformierten Weiterbildungsförderung. ${ }^{13}$ Im dreizehnten Kapitel, das „Sonderregelungen“ enthält, ist die Vorschrift über den Vermittlungsgutschein versteckt, der die Vermittlung durch private Vermittler fördern soll. ${ }^{14}$ Geregelt sind diese neuen Erscheinungsformen des Leistungserbringungsrechts in Form mühsam aufzufindender Besonderheiten einzelner Leistungsansprüche. Das sind sozusagen normative Inseln, auf denen sich leistungserbringungsrechtliche Strukturen in der Weite ihres Besonderen Teils eher verbergen, wie etwa die besagten Regeln der Weiterbildungsförderung im SGB III.

Ein abschreckendes Beispiel für eine verständnishinderliche Regelungstechnik bietet die Vorschrift des § 16 SGB II über die von der Agentur für Arbeit - ggf. durch Dritte $\mathrm{zu}$ erbringenden Eingliederungsleistungen.

3. Einen weiteren Befund bietet schließlich das SGB IX als Querschnittsgesetzbuch. Dieses Gesetz bemüht sich um die Kodifizierung gemeinsamer Regeln für acht unterschiedliche Träger der Leistungen zur Teilhabe. Mit Blick auf Fragen der Leistungserbringung ist das zweite Kapitel des ersten Teils von Bedeutung, also die Regelungen über die Ausführung von Leistungen zur Teilhabe. Interessanterweise finden sich dort einige Vorschriften, die sich in allgemeiner Form mit den Beziehungen der Rehabilitationsträger zu den Rehabilitationsdiensten- und Einrichtungen befassen. U. a. gibt es dort einen $\S 21$ über die Ausgestaltung von Verträgen mit den Leistungserbringern. ${ }^{15}$ Anders als bei der soeben dargestellten leistungserbringungsrechtlichen Parallelgesetzgebung hat sich der Gesetzgeber an dieser Stelle in einem emphatischeren Sinne kodifikatorisch angestrengt.

$13 \S 77$ ff. SGB III i. d. F. des Ersten Gesetzes für moderne Dienstleistungen am Arbeitsmarkt vom 23.12.2002, BGB1. I S. 4607 und dazu BT-Drs. 15/25, S. 29; dazu Hänlein, Governance und Arbeitsmarkt, RsDE 65 (2007), S. 43 ff. (57-60); eingehend demnächst Roos, Die Akkreditierung fachkundiger Stellen und Zertifizierung für Träger von Maßnahmen der beruflichen Weiterbildung im System der Qualitätssicherung nach den $\S \S 77$ ff. SGB III - Rechtliche Probleme und mögliche Lösungen für die betriebliche Praxis, Diss., Kassel 2007.

$14 \S 421 \mathrm{~g}$ SGB III zum Vermittlungsgutschein, eingeführt durch das Gesetz zur Vereinfachung der Wahl der Arbeitnehmervertreter in den Aufsichtsrat vom 23.3.2002, BGB1. I S. 1130; dazu Hänlein (Fußn. 13), RsDE 65 (2007), S. 48-51.

15 Dazu BT-Drs. 14/5074, S. $104 \mathrm{f}$. 
III.

Will man zusammenfassend den kodifikatorischen Zustand des Leistungserbringungsrechts beschreiben, muss man wohl sagen, dass es etwas Licht, aber auch ziemlich viel Schatten gibt. Was folgt daraus, insbesondere für die Sozialrechtswissenschaft?

1. Eine Sozialrechtswissenschaft, die sich um die Dogmatik des Sozialrechts bemüht, erfüllt unterschiedliche Aufgaben. Ihre Anstrengungen um Systematisierung, um deutende und differenzierende Darstellung des geltenden positiven Rechts, zielen zunächst darauf ab, die Masse des Stoffs der Lehre zugänglich zu machen. Dementsprechend finden sich in den vorliegenden Lehrbüchern auch Darstellungen des Leistungserbringungsrechts, die sich meist in die Darstellungen der einzelnen Sicherungszweige, vornehmlich des Rechts der gesetzlichen Krankenversicherung, aber auch der sozialen Pflegeversicherung einfügen. ${ }^{16}$

Zum zweiten haben rechtsdogmatische Bemühungen die Praxis der Rechtsanwendung im Blick, insbesondere die richterliche Praxis. Insoweit geht es darum, die Reflexion und Kommunikation über vorstellbare, anhängige oder bereits entschiedene Anwendungsfragen des geltenden Rechts zu erleichtern und anzureichern. ${ }^{17}$

Schließlich haben sozialrechtsdogmatische Bemühungen neben den erwähnten rechtsdidaktischen und rechtspraktischen Funktionen auch eine rechtsstaatliche und rechtspolitische Funktion. Ohne die Formulierung rechtlicher Prinzipien, ohne die Arbeit an System und Begriffen des Rechts, ohne die Konstruktion und Konkretisierung rechtlicher Institute wird es schwerlich ein stimmiges, ein transparentes, ein gutes Gesetz geben können. Deshalb sollte auch im Vorfeld von größeren Gesetzgebungsprojekten das institutionalisierte Gespräch mit der Rechtswissenschaft gepflegt werden, wie es etwa bei der Erarbeitung des Entwurfs des SGB I geschehen ist. ${ }^{18}$ Ist ein Gesetz einmal beschlossen, hat sich jedoch die rechtspolitische Aufgabe der Wissenschaft keineswegs erledigt. Vielmehr zeigt gerade die Entwicklung der Kodifikation des Sozialrechts sehr deutlich, dass die mannigfachen tagesaktuellen Bedürfnissen entspringenden Gesetzesänderungen immer wieder zu Widersprüchen und Systembrüchen führen, die sogar ver-

16 Beispiele: Bley/Kreikebohm/Marschner, Sozialrecht, 9. Aufl. Neuwied 2007, Rdnr. 655 ff. betr. Krankenversicherung; Eichenhofer, Sozialrecht, 6. Aufl. Tübingen 2007, S. 203-211 betr. Krankenversicherung; Fuchs/Preis, Sozialversicherungsrecht, Köln 2005, S. 318-342 betr. Krankenversicherung und S. 404-428 betr. Pflegeversicherung; Igl/Welti, Sozialrecht, 8. Aufl. Neuwied 2007, S. 110-116 betr. Krankenversicherung, S. 135-137 betr. Pflegeversicherung, S. 299 betr. Sozialhilfe und S. 355 f. betr. SGB IX; Waltermann, Sozialrecht, 7. Aufl. Heidelberg 2008, S. 98-109 betr. Krankenversicherung und S. 120-123 betr. Pflegeversicherung.

17 Vgl. auch Hassemer, Rechtssystem und Kodifikation, in: Kaufmann/Hassemer/Neumann (Hrsg.), Einführung in die Rechtsphilosophie und Rechtstheorie der Gegenwart, 7. Aufl. Heidelberg 2004, S. 251 ff. (255 f. und 264).

18 Vgl. Zacher, Das Vorhaben des Sozialgesetzbuches, Percha 1973, S. 16. 
fassungsrechtliche Bedeutung erlangen können. ${ }^{19}$ So gesehen stellt sich der Wissenschaft Kodifikationspflege als Daueraufgabe, wie es Ursula Köbl ausgedrückt hat. ${ }^{20}$ Diese Aufgabe stellt sich in besonderer Schärfe im Hinblick auf das Leistungserbringungsrecht.

2. Es soll hier nicht eine abstrakte Forderung aufgestellt, sondern vor allem darauf hingewiesen werden, dass die Forderung nach kodifikatorischer Verbesserung im Bereich des Leistungserbringungsrechts einem dringenden Bedürfnis der Praxis entspricht. Bereits vor einigen Jahren hatte Schellhorn auf einer Freiburger Tagung beklagt, dass es in SGB XI, BSHG und SGB VIII differenzierte Leistungserbringungsrechte mit ausgeprägtem Vertragswesen und Schiedsstellenverfahren gibt, die im einzelnen nicht aufeinander abgestimmt sind und widersprüchliche Regelungen enthalten; als Hintergrund dieser unzuträglichen Parallelkodifikation identifizierte er unterschiedliche ministerielle Zuständigkeiten; vor allem aber fehle es an ausreichenden vorparlamentarischen Vorarbeiten zu einem einheitlichen und klar konzipierten Vertragsrecht der Leistungserbringung. ${ }^{21}$ Diese Forderung ist inzwischen lauter geworden. Vor zwei Jahren haben sich die „Vorsitzenden und Geschäftstellenleiter/innen der Schiedsstellen nach § 76 SGB XI und $\S 80$ SGB XII“ zu Wort gemeldet und Vorschläge für eine verbesserte rechtliche Ausgestaltung der sozialrechtlichen Schiedsstellen vorgelegt ${ }^{22}$, wobei die präsentierten Vorschläge deutlich über technische Aspekte der Schiedsstellen hinausgehen. Vielmehr lässt man sich von den kodifikatorischen Errungenschaften in SGB V und SGB IX inspirieren und schlägt die Einführung neuer Regelungen in die allgemeinen Teile vor. Insbesondere wird vorgeschlagen, neue Vorschriften - als $\S \S 97 a-97 d$ - in das Dritte Kapitel des SGB X aufzunehmen. ${ }^{23} \S 97$ a über die Ausführung von Sozialleistungen durch Dritte soll in Anlehnung an $\S 69$ SGB V formuliert werden. § 97b SGB X über Qualität und Wirtschaftlichkeit soll allgemeine Vorschriften über Rahmenvereinbarungen auf Landesebene sowie über einrichtungsindividuelle Vereinbarungen enthalten. Sodann sollen in Form neuer $\S \S 97 \mathrm{c}$ und d SGB X einheitliche Regelungen über sozialrechtliche Schiedsstellen und deren Verfahren und Besetzung eingeführt werden.

19 Zum rechtsstaatlichen Gebot der Normenklarheit etwa BVerfGE 108, 52, 75 betr. Nichtanrechnung von Kindergeld auf Unterhaltsanspruch des Kindes.

$20 \mathrm{Köbl}$, Kodifikation - nicht nur bei Gelegenheit dringlicher Sachreform!, in: Ruland/von Maydell/Papier (Hrsg.), Verfassung, Theorie und Praxis des Sozialstaats, Festschrift für Hans F. Zacher zum 70. Geburtstag, Heidelberg 1998, S. 389 ff. (403 ff.); Zacher hat eine Institution mit unabhängigen Sachverständigen zur Verbesserung der inneren Stimmigkeit der Kodifikation gefordert; $\mathrm{Za}$ cher (Fußn. 2), RsDE 47 (2002), S. 1 ff. (27 f.).

21 Vgl. den Bericht bei Hänlein, in: Köbl/Brünner, Die Vergütung von Einrichtungen und Diensten nach SGB XI und BSHG, Baden-Baden 2001, S. 94 ff. (101) = ZfSH/SGB 2001, S. 331 ff. (335).

22 NDV 2006, S. 302 ff.

23 In diese Richtung bereits Hänlein, in: Köbl/Brünner (Fußn. 21), S. 94 ff. (101, Fußn. 33) = ZfSH/SGB 2001, S. 331 ff. (335, Fußn. 35). 
Diese Forderungen haben gerade jüngst, und zwar erneut aus der Praxis, vehemente Unterstützung erhalten. ${ }^{24}$ Mir scheint es sinnvoll, in diese Richtung auch wissenschaftlich weiter nachzudenken. Es kann hier an das Zachersche dictum erinnert werden, dass der „, Sinn der Kodifikation (darin liege), die Teile auf ein besseres Ganzes hin zu transzendieren" 25 , eine gerade auch mit Blick auf den kodifikatorischen Zustand des Leistungserbringungsrechts ernst zu nehmende Mahnung.

24 Griep, Entbürokratisierung des sozialrechtlichen Leistungserbringungsrechts, RsDE 66 (2008), S. $27 \mathrm{ff}$.

25 Zacher (Fußn. 2), RsDE 47 (2002), S. 1 ff. (27). 


\section{Diskussionsbericht}

I. Ein Schwerpunkt der Diskussion, die an die Beiträge zum Themenblock „Das Leistungserbringungsverhältnis“ mit den Referaten von Fuchs, Trenk-Hinterberger und Hänlein anschloß, widmete sich der Bedeutung und den Aufgaben der Sozialrechtsdogmatik und griff damit ein zentrales Thema des Symposiums auf. Die grundlegende Funktion rechtsdogmatischer Forschung bestehe darin, die gedanklichen und strukturellen Ordnungen, auf denen das positive Recht aufbaue, sichtbar zu machen, um diese als Basis für das Verstehen und die Anwendung des Rechts nutzbar machen zu können. Gleichzeitig helfe die Dogmatik auch, Fragestellungen der Lebenswirklichkeit aufzuzeigen, auf die das geltende Recht bislang keine Antwort gefunden habe. Damit diene es der Ermittlung des Bedarfs für die Weiterentwicklung des Rechts, gebe hierfür aber zugleich auch bestimmte Leitlinien vor.

Diese grundlegende Bedeutung der Rechtsdogmatik für die Fortentwicklung des Rechts wurde durch Ausführungen über die Bemühungen um die Neuordnung des österreichischen Gesundheitswesens untermauert. Die momentan herrschenden politischen Machtverhältnisse hätten zu Lösungsvorschlägen geführt, die die bestehenden Strukturen aus Gesamt- und Einzelverträgen, die den Ausgleich verschiedener Interessen gewährleisten, erodieren ließen. Nur die dogmatische Auseinandersetzung mit den grundlegenden Systemfragen lasse dies deutlich werden.

Demgegenüber wurde angemahnt, man müsse sich auch der Grenzen des mit Rechtsdogmatik und mit Rechtsvergleichen zu erzielenden Erkenntnisgewinns bewußt sein. Die Entwicklung von rechtlichen Lösungen für bestimmte soziale Probleme könne nur dann erfolgreich sein, wenn sie nicht isoliert auf rechtsdogmatischen und rechtsvergleichenden Erwägungen aufbaue, sondern auch die Änderungen der wirtschaftlichen und gesellschaftlichen Rahmenbedingungen auf nationaler und internationaler Ebene in den Blick nehme. Zudem verbiete die multifunktionale Ausgestaltung sozialrechtlicher Systeme, monokausale Aussagen über die Effektivität bestimmter Regelungsstrukturen zu treffen.

Von anderer Seite wurde darauf verwiesen, daß die rechtsdogmatische Forschung mit erheblichen Defiziten in der Rechtstatsachenforschung konfrontiert sei, d. h. im $\mathrm{Zu}-$ sammenhang mit der Frage, ob und inwieweit die Regelungen, die den Gegenstand rechtsdogmatischer Überlegungen bilden, tatsächlich umgesetzt würden.

Die wissenschaftliche Auseinandersetzung mit der Eingliederungsvereinbarung oder mit dem trägerübergreifenden Persönlichen Budget etwa zeige, daß dieses Defizit leicht dazu führen könne, daß sich verschiedene persönliche Erfahrungen mit der Realität der Rechtsanwendung in unterschiedlichen rechtsdogmatischen Schlüssen ausdrückten.

II. Einen weiteren Hauptpunkt der Diskussion bildeten Beiträge zu verschiedenen Aspekten der Kostenerstattung und des Persönlichen Budgets, mit denen sich Trenk- 